\title{
Efficient Priority based Multipath Routing in Wireless Sensor Network for Multimedia Streaming
}

\author{
Vairam T. \\ PSG College of Technology \\ India
}

\author{
Kalaiarasan C. \\ SNS College of Technology \\ India
}

\begin{abstract}
Monitoring the target area lively by video sensor node will improve the ability of event description. Since the sensor nodes have limited capacity, single path routing does not suite to transfer video content. Accordingly there is a need for multipath routing for efficient transmission of video content. However not all the path in multipath routing is suited for video transmission because one or few path may not have sufficient energy to transfer the video stream, but it can able to transfer data stream. Based on the application, the type of data either video stream or data stream plays a different role. Significance levels are different for each of its data type. Priority for the data is to be given based on their significance level. In this paper, a novel approach called Efficient Priority Based Multipath Routing (EPBMR) is proposed which optimizes the multiple disjoint path found from Cost Based Multipath Routing (CBMR) by giving priority to the cost of each disjoint path and type of data. The results show that EPBMR can able to choose maximum number of path based on the priority.
\end{abstract}

\section{General Terms}

Multipath Routing, Disjoint Path

\section{Keywords}

path cost, CBMR, End-to-End Delay

\section{INTRODUCTION}

Nowadays the adoption of wireless sensor networks in day to day life has been increased. The importance of sensor device plays a major role in application such as area monitoring, health care monitoring, forest fire detection, landslide detection, water quality monitoring and natural disaster prevention etc. Since the sensor devices are tiny in size and very less cost, nearly thousand to million nodes can be deployed in particular area. The main responsibility of the sensor nodes in each above mentioned application is to sense the environmental changes or an unexpected event occurred in target area and sends the sensed data to sink node /Base Station (BS). Resource limitations of the sensor node and unreliability of low power wireless links in combination with various performance demands of different applications impose many challenges in designing an efficient communication protocols for wireless sensor networks.

Routing is the one important issue to be considered to fulfill different performance demands of various applications in Wireless Sensor Network. The latency, throughput, energy utilization, and network life time are the main consideration for the most proposed protocols. However, the attributes of sensed data may vary depending on type of application; it may be a delay sensitive or reliability demanding data. For example, the sensor network that is used for monitoring the normal temperature in weather monitoring station will not require receiving the sensed data within certain time limit. On the other hand, the sensor network used for fire detection in a forest, the sensed data to be reached on time to the processing centre. In addition, the opening of multimedia sensor devices along with the increasing attention in real time applications has made stringent constraints on both throughput and delay. Single path routing does not suite to transfer video content because the same set of nodes are not used in a path to transfer the video content as the size is very large. Accordingly there is a need for multipath routing for efficient transmission of video content. However not all the path in multipath routing is suited for video transmission because one or few path may not have sufficient energy to transfer the video stream, but it can able to transfer data stream. Based on the application, the type of data either video stream or data stream plays a different role. Significance levels are different for each of its data type. Priority for the data is to be given based on their significance level. The objective of this work is to provide a better utilization of network resources and extend the network life time by providing the priority for choosing the best path for transferring the video stream and data stream.

Work presented here is organized as follows: Section 2 presents the related work with respect to multipath routing and multimedia data. Section 3 presents a description of the proposed system. Section 4 discusses implementation and the result analysis. Finally, the conclusion is provided on Section 5 .

\section{RELATED WORK}

Multipath routing in WSN is comparatively a new research topic along with other research topic in WSN such as localization, cross layer interaction etc. Enormous surveys have been done on Multipath routing in WSN. [1] Comparison of routing protocols has been made. The routing protocols such as DSR, AODV and MCP-DE are considered for comparison. These protocols are evaluated based on data delivery ration and delay. From the results MCP-DE outperforms than AODV and DSR. MCP-DE is one among the multiple disjoint protocols. [2] Surveys illustrate that transferring multimedia data in WSN could also be considered as research topic in WSN. [3] Gave survey work on multimedia communication in WSN and narrated mobile multimedia and WSN fields in application, transport and network layer. Most the existing work which are being carried out are concentrating in either increasing throughput and reducing the delay by considering the QoS parameter. [4] Proposed MPMPS routing approach, which divide the video stream as image stream and audio stream and provides either low priority or high priority for image stream and audio stream. Based on the priority the suitable disjoint path has been selected among all disjoint paths which are actually obtained using TPGF [5]. MPMPS consider only video streaming but there is a need for data stream based on the application and the situation. In this paper it address both video stream and data stream and provides priority to the type 
data whether it may be video stream or data stream. Cost Based Multipath Routing (CBMR) [6] is been used for finding disjoint paths between source and destination.

\section{PROPOSED WORK}

\subsection{Network Model}

In this proposed work the heterogeneous wireless sensor network which consists of both video sensor network and data sensor network is considered. Video sensor network is responsible for capturing the event in a multimedia form and a data sensor device is responsible for monitoring the event. All nodes are static in their position. Each node knows its own position and their neighbor`s position. All nodes have to calculate their success rate for sending and receiving the data.

$$
\operatorname{Success}\left(\operatorname{Node}_{i}\right)=\frac{1}{S s * S r}
$$

Where " $i$ " represents the node number. "Ss" is probability of successfully sending rate and " $\mathrm{Sr}$ " is the probability of successfully receiving rate

\subsection{Priority Parameters}

Two parameters are consider for priority namely path cost and data type. The CBMR is used for finding the different disjoint path between source node and sink node.

$D P=\left\{D P_{1}, D P_{2}, D P_{3}, \ldots . D P_{n}\right\}$

Where DP is a set of Disjoint Path between the source node and the sink node/Base Station obtained from CBMR. DP consists of set of nodes through which the data is transferred from source node to sink node. Node Cost is calculated for each node in $\{D P\}$

$$
\text { NodeCost }_{i}=\operatorname{success}\left(\text { Node }_{i}\right) \cdot \frac{1}{\text { Residual Energy Level }}
$$

Path cost is calculated as follows

$$
\text { PathCost }_{j}=\sum_{i=1}^{N} \text { NodeCost }_{i}+N
$$

Pathcost is computed for each disjoint path $\mathrm{j}$. i represent the $\mathrm{i}^{\text {th }}$ node in a disjoint path and $\mathrm{N}$ is the number of hop count for a particular $\mathrm{DP}_{\mathrm{j}}$

The second priority parameter is Data type. Data type is one of the important parameter to be considered for priority. Depends on the situation, the requirement of data would be differ. In application like traffic monitoring, if there is an accident in a monitoring field, intimation should reach the base station immediately for further action. Here text data plays a major role rather than image file. Hence priority is to be given to text data. In fire detection monitoring application, image file plays major role to send a details about how badly the field was affected due to fire in the form image. Hence priority should be given to image file. In some application, both forms of data takes equal priority, in such a case the data can be split into image stream and data stream. Image stream will be given high priority since it consumes more energy to transfer due to they are large in size. Data stream will be given low priority. Based on the priority the disjoint path will be assigned for the data. Always the high priority or low priority data will be assigned with the DP which has maximum or minimum of path cost respectively.

$$
\begin{aligned}
& L P T P=\operatorname{Min}\left(\text { PathCost }\left\{D P_{1}, D P_{2}, D P_{3}, \ldots . D P_{n}\right\}\right) \\
& H P T P=\operatorname{Max}\left(P a t h \operatorname{Cost}\left\{D P_{1}, D P_{2}, D P_{3}, \ldots . D P_{n}\right\}\right)
\end{aligned}
$$

A set of Low Priority Transmission Path (LPTP) is chosen which has minimum path cost. A set of High Priority Transmission Path (HPTP) is chosen which maximum path has cost. The number of maximum path and the minimum are decided by Transmission Cost and size of data.

\subsection{Work Flow of EPBMR}

Figure 1 shows the workflow of EPBMR. Initially the number of disjoint path which are obtained from the CBMR is considered as input to the EPBMR. The priority is assigned for the data according to the situation. As mentioned in section 3.2 , based on the application the priority will be different. The path cost is calculated using equation (4). Transmission cost is assigned for each disjoint path. Transmission cost is a threshold hold value which is used to determine the transmission capacity of each disjoint path. The disjoint paths are ordered either in ascending manner or descending manner based on the priority given to the type of data. The disjoint path will be chosen by considering size of the data, transmission capacity $(\mathrm{Tc})$ of disjoint path and priority of the data type.

\section{PERFORMATION EVALUATION}

The EPBMR is simulated in MATLAB environment. For this simulation, the network area size of $1000 \times 1000 \mathrm{~m}$ with low density (50 Nodes) and high density (200 Nodes) of sensor nodes are considered. The transmission rage for low density and high density are is $100 \mathrm{~m}$ and $50 \mathrm{~m}$ respectively. All nodes are randomly deployed in the network field with randomly generated energy. The source node and the sink node can be decided dynamically.

If an event is detected in the target area, The CBMR techniques is being executed for identifying different disjoint paths from source node to sink node. Figure 3 and Figure 4 shows the paths identified for low priority transmission path which are denoted in solid red line. High priority transmission path are denoted in dotted blue line. Figure 3 and Figure 4 represents low density and high density respectively. The performance of the EPBMR is been compared with CBMR which does not provide any priority for the data as well as for the path cost. This simulation is done for the video of avi file format which contains 24 frames per second and average frame size is $20 \mathrm{~K}$. The path chosen by EPBMR and CBMR is tabulated in Table 1 and Table 2 for low density and high density correspondingly. The symbol $\sqrt{ }$ in a field indicates the corresponding path has been chosen for transmission. The Table 1 and Table 2 also list the Average End-to-End delay of CBMR and EPBMR and it is inferred that End-to-End delay is reduced in EPBMR when compared with CBMR. 


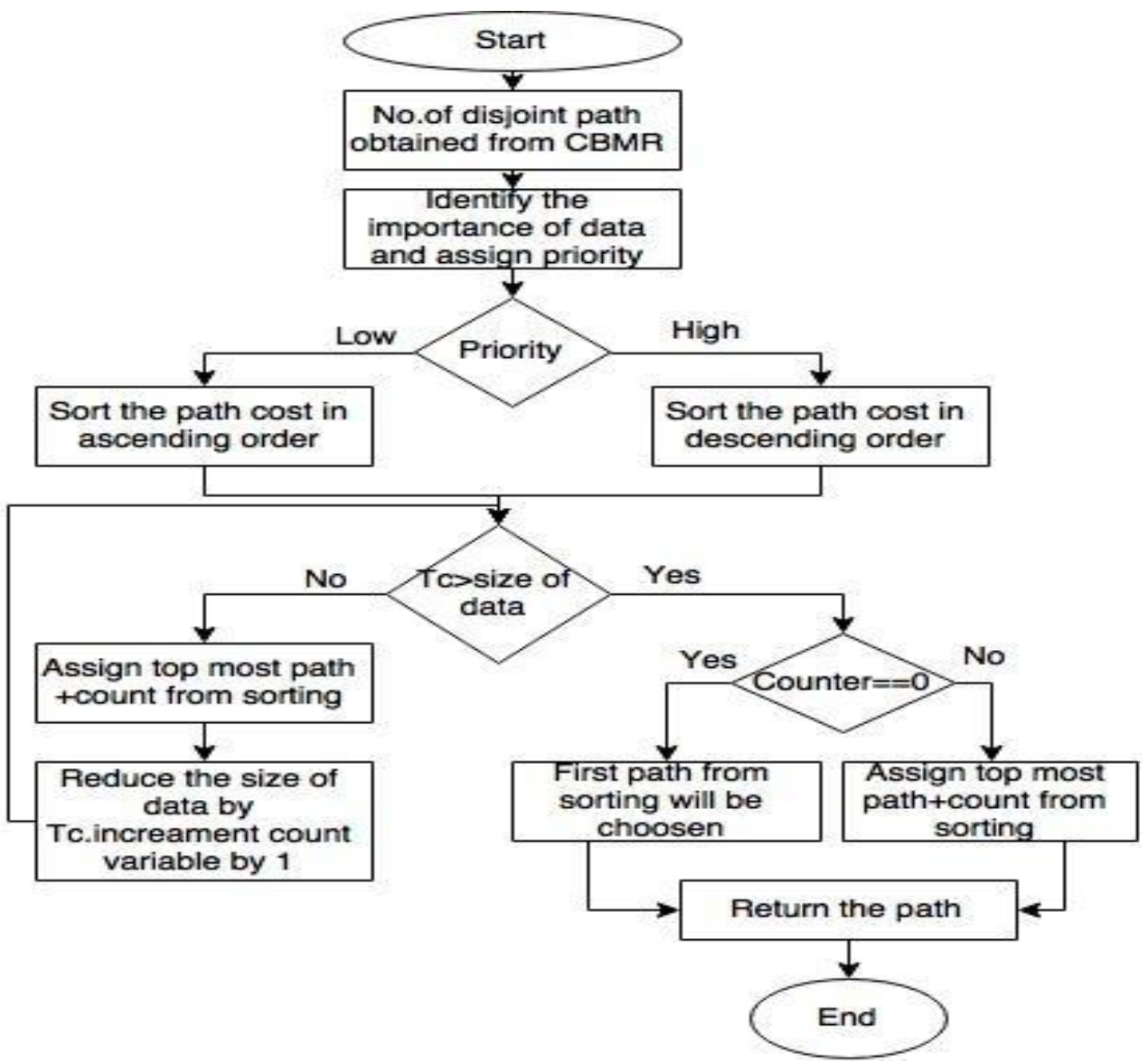

Fig 1: Work Flow of EPBMR

Table 1 End-to-End Delay for High Density

\begin{tabular}{|c|c|c|c|c|c|c|}
\hline \multirow{2}{*}{$\begin{array}{c}\text { No. } \\
\text { of } \\
\text { Disjo } \\
\text { int } \\
\text { Path }\end{array}$} & \multicolumn{2}{|c|}{$\begin{array}{l}\text { High Priority } \\
\text { for Video } \\
\text { Stream }\end{array}$} & \multicolumn{2}{|c|}{$\begin{array}{l}\text { High Priority } \\
\text { for Data } \\
\text { Stream }\end{array}$} & \multicolumn{2}{|c|}{$\begin{array}{l}\text { Average End- } \\
\text { to-End delay } \\
\text { in milli second }\end{array}$} \\
\hline & $\begin{array}{l}\text { CB } \\
\text { MR }\end{array}$ & $\begin{array}{l}\text { EPB } \\
\text { MR }\end{array}$ & $\begin{array}{l}\text { CB } \\
\text { MR }\end{array}$ & $\begin{array}{l}\text { EPB } \\
\text { MR }\end{array}$ & $\begin{array}{c}\mathbf{C B M} \\
\mathbf{R}\end{array}$ & $\begin{array}{l}\text { EPB } \\
\text { MR }\end{array}$ \\
\hline Path1 & $\sqrt{ }$ & & $\sqrt{ }$ & & \multirow{4}{*}{$\begin{array}{c}544.2 \\
81\end{array}$} & \multirow{4}{*}{$\begin{array}{c}522.0 \\
5\end{array}$} \\
\hline Path2 & $\sqrt{ }$ & $\sqrt{ }$ & $\sqrt{ }$ & & & \\
\hline Path3 & $\sqrt{ }$ & $\sqrt{ }$ & $\sqrt{ }$ & $\sqrt{ }$ & & \\
\hline Path4 & $\sqrt{ }$ & & $\sqrt{ }$ & & & \\
\hline
\end{tabular}

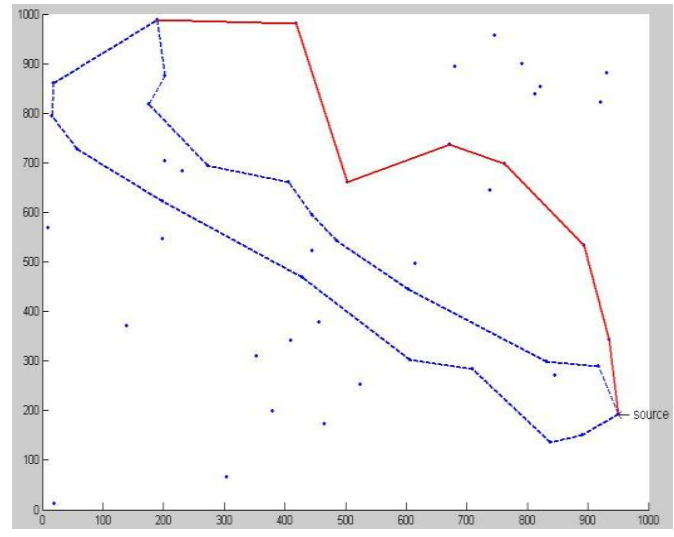

Table 1 End-to-End Delay for Low Density

\begin{tabular}{|c|c|c|c|c|c|c|}
\hline \multirow{2}{*}{$\begin{array}{c}\text { No. } \\
\text { of } \\
\text { Disjoi } \\
\text { nt } \\
\text { Path }\end{array}$} & \multicolumn{2}{|c|}{$\begin{array}{l}\text { High Priority } \\
\text { for Video } \\
\text { Stream }\end{array}$} & \multicolumn{2}{|c|}{$\begin{array}{l}\text { High Priority } \\
\text { for Data } \\
\text { Stream }\end{array}$} & \multicolumn{2}{|c|}{$\begin{array}{l}\text { Average End- } \\
\text { to-End delay } \\
\text { in milli } \\
\text { second }\end{array}$} \\
\hline & $\begin{array}{l}\text { CB } \\
\text { MR }\end{array}$ & $\begin{array}{l}\text { EPB } \\
\text { MR }\end{array}$ & $\begin{array}{l}\text { CB } \\
\text { MR }\end{array}$ & $\begin{array}{l}\text { EPB } \\
\text { MR }\end{array}$ & $\begin{array}{l}\text { CB } \\
\text { MR }\end{array}$ & $\begin{array}{l}\text { EPB } \\
\text { MR }\end{array}$ \\
\hline Path1 & $\sqrt{ }$ & $\sqrt{ }$ & $\sqrt{ }$ & $\sqrt{ }$ & \multirow{3}{*}{$\begin{array}{c}289.5 \\
2\end{array}$} & \multirow{3}{*}{$\begin{array}{c}253.9 \\
21\end{array}$} \\
\hline Path2 & $\sqrt{ }$ & & $\sqrt{ }$ & & & \\
\hline Path3 & $\sqrt{ }$ & $\sqrt{ }$ & $\sqrt{ }$ & & & \\
\hline
\end{tabular}

Figure 3 Disjoint path for Low Density

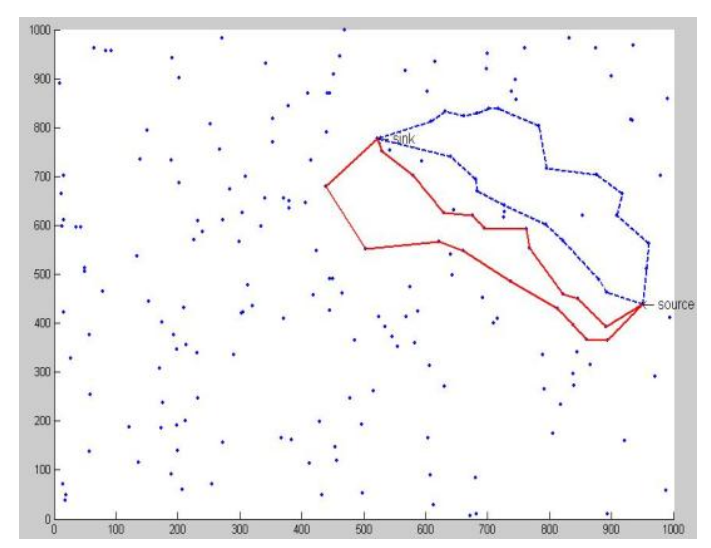

Figure 4 Disjoint path for High Density 
Energy is one of the QoS parameter to be considered for evaluating the performance of routing algorithms. Hence energy has been considered as one of the performance parameter.

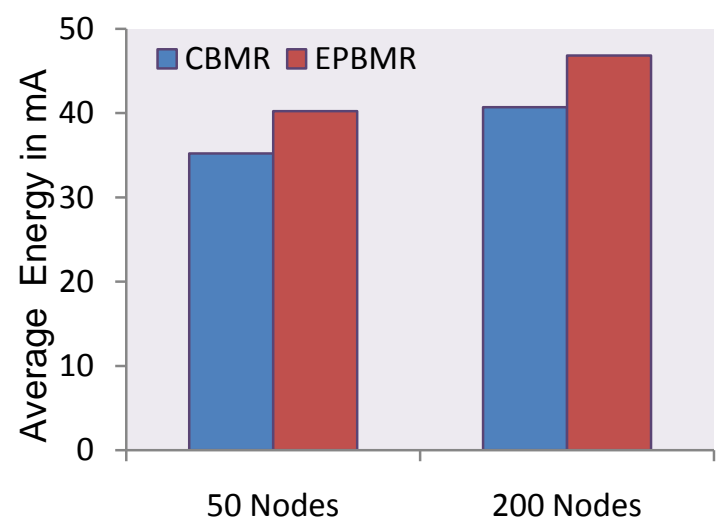

Figure 5 Average Residual Energy

After sending the data to the sink node, the average energy is calculated by considering the residual energy of all nodes in network. From Figure 5 It is inferred that the average remaining energy is more for EPBMR when compared to CBMR. Since, EPBMR involves less number of nodes in transferring the data from source node to sink node.

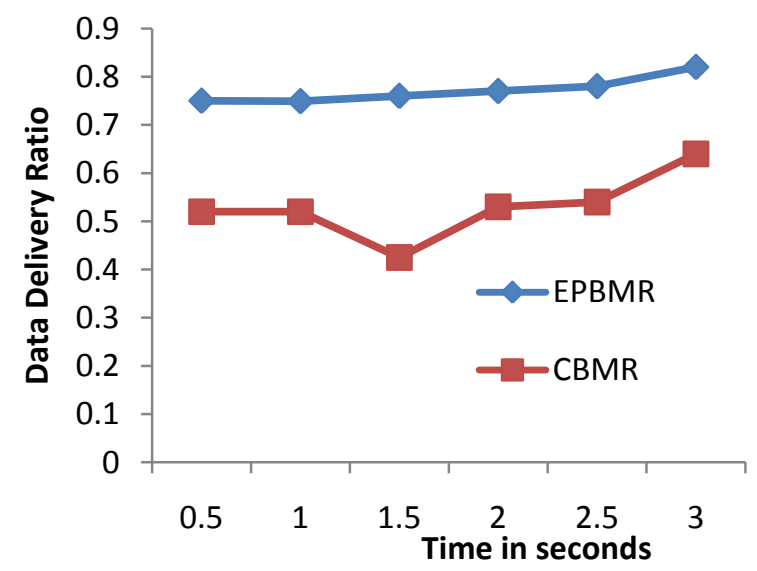

Figure 6 Data Delivery Ratio for High Density

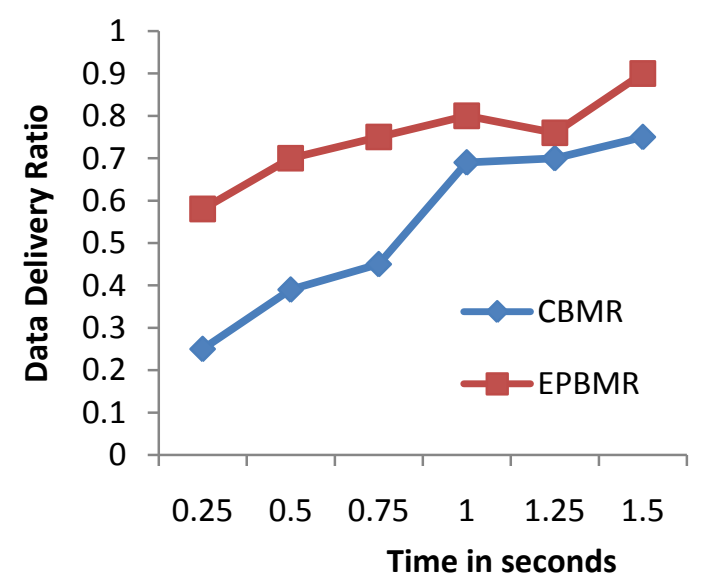

Figure 7 Data Delivery Ratio for Low Density

The data delivery ratio was calculated between the number of data packets that are sent by the source and the number of data packets that are received by the sink. Data delivery ratio indicates both the loss ratio of the routing protocol and the effort required to receive the data.

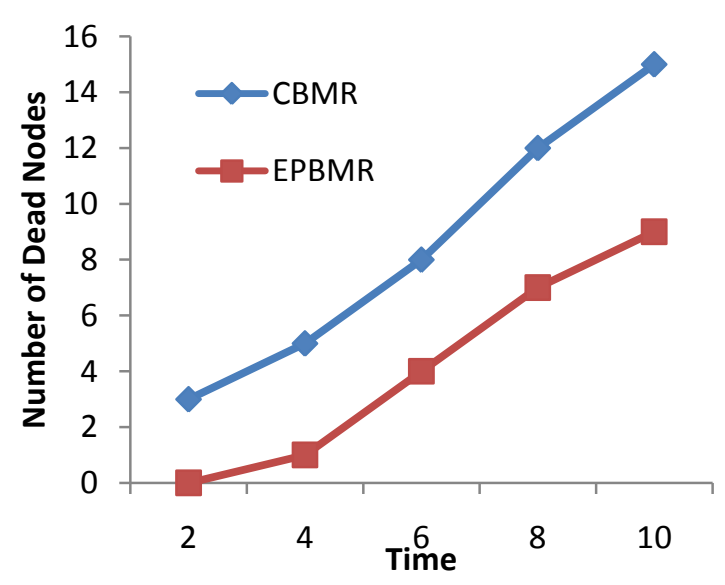

Figure 8 Number of Dead Nodes for High Density

The Figure 6 and Figure 7 show the data delivery ratio of CBMR and EPBMR for high density and low density respectively. From the results it is inferred that the data deliver ratio of EPBMR is good than CBMR. This is because EPBMR chose the path which has high cost. Figure 8 shows the number of dead node between CBMR and EPBMR. From the figure 8 it is inferred that the number of dead nodes in EPBMR is less than the CBMR.

\section{CONCLUSION}

In this paper, a novel approach called Efficient Priority Based Multipath Routing (EPBMR) has been implemented which optimizes the multiple disjoint path found from Cost Based Multipath Routing (CBMR) by giving priority to the cost of each disjoint path and type of data. The significance level of data is identified, based on that this approach selects set of Low Priority Transmission Path (LPTP) and High Priority Transmission Path (HPTP) for transferring the data from source node to Sink node. Since only limited number of disjoint path is selected for transmission, the End-to-End delay is reduced and the residual energy is increased when compared to CBMR. Reducing the End-to-End delay and increasing average residual energy will help in extending the network lifetime and enabling the better utilization of network resources.

\section{REFERENCES}

[1] Yamuna Devi, Vairam T, Kalaiarasan C, 2012, "Efficient Comparison of Multipath Routing Protocols in WSN", Proceedings of International Conference on Computing, Electronics and Electrical Technologies.

[2] Akyildz I E, Melodia T, Chowdhury K R, 2007, “ A survey on Wireless Multimedia Sensor Networks", Computer Networks, Vol 51, Issue 4 pp 921-960.

[3] Gurse E, Akan O B, 2005, "Multimedia Communication in Wireless Sensor Networks" Annals of Telecommunication, Vol 60, no 7-8 pp 799-827.

[4] Lin Zhang, Manfred Hauswirth et al. , 2008, “ Multipriority Multipath Selection for Video streaming in Wireless Multimedia Sensor Networks", Ubiquitos Intellegence and Computing, Lecture notes in computer Science, Volume 5061, pp 439-452. 
[5] Shu L, Zhou Z B et al, 2007, “ Transmitting Streaming Data in Wireless Multimedia Sensor Networks with Holes", in Proceedings of the sixth international Conference on Mobile and Ubiquitous Multimedia.

[6] Vairam T, Kalaiarasan C, 2015, "Cost based Multipath Routing in WSN for Multimedia data" ARPN journal of Engineering and Applied Science.

[7] Zijian Wang, Eyuphan Bulut, and Boleslaw K.Szymanski, 2009, "Energy Efficient Collision Aware Multipath Routing for Wireless Sensor Networks", In Proc. International Conference on Communication, pp.1-5.

[8] Y. Wang and S.Lin, 2001, "Error resilient Video Coding using Multiple Description motion compensation", In Proc. IEEE Fourth Workshop on Multimedia Signal Processing, pp.441-446.

[9] MarjanRadi, BehnamDezfouli, Kamalurlnizam Abu Bakar, ShukorAbdRazak and Mohammad Ali Nematbakhsh, 2011, "Interference-aware multipath routing protocol for Qos improvement in event-driven wireless sensor networks", In Proc Tsinghua Science and Technology, Vol.16, No.5.

[10] Jonathan L. Bredin, Erik D. Demaine, Mohammad Taghi Hajiaghayi, and Daniela Rus, 2010, "Deploying Sensor Networks With Guaranteed Fault Tolerance", In Proc IEEE/ACM Transactions on Networking, Vol.18, NO.1.
[11] Bettstetter C, Reseta G, Santi P, 2003, “ The node distribution of the random way point mobility model for wireless ad hoc networks", IEEE Transactions on Mobile Computing 2(3), 257-269.

[12] S. Lindsey, C.S. Raghavendra and K.M Sivalingam, 2002, "Data Gathering algorithms in sensor networks using energy metrics", IEEE Transaction on Parallel and Distributed Systems, Vol . 13, no.9, pp.924-935.

[13] Shuang Li, Raghu KisoreNeelisetti, Cong Liu and Alvin Lim, 2010, "Efficient Multipath protocol for Wireless Sensor Networks", In Proc International Journal of Wireless and Mobile Networks (IJWMN), Vol.2, No.1.

[14] Akkava K and Younis, M, 2005, “A Survey on Routing Protocols for Wireless Sensor Networks", In Proc. Ad Hoc netw.J. Vol.3, pp.325-349.

[15] Kai Lin , Rodrigues, J.J.P.C., Hongwei Ge , Naixue Xiong, 2011, "Energy Efficiency QoS Assurance Routing in Wireless Multimedia Sensor Networks", Systems Journal, IEEE Volume:5 issue:4.

[16] C.E Perkins and E.M Royer, 1999, "Ad Hoc OnDemand Distance Vector Routing”, Proc. Second IEEE Workshop Mobile Computing Systems and Application, pp.90-100. 